\title{
Seasonality of acquisition of respiratory bacterial pathogens in young children with cystic fibrosis
}

Kevin J. Psoter ${ }^{*}$, Anneclaire J. De Roos ${ }^{2}$, Jon Wakefield ${ }^{3}$, Jonathan D. Mayer ${ }^{4}$ and Margaret Rosenfeld ${ }^{5,6}$

\begin{abstract}
Background: Seasonal variations are often observed for respiratory tract infections; however, limited information is available regarding seasonal patterns of acquisition of common cystic fibrosis (CF)-related respiratory pathogens. We previously reported differential seasonal acquisition of Pseudomonas aeruginosa in young children with CF and no such variation for methicillin-susceptible Staphylococcus aureus acquisition. The purpose of this study was to describe and compare the seasonal incidence of acquisition of other respiratory bacterial pathogens in young children with CF.

Methods: We conducted a retrospective study to describe and compare the seasonal incidence of methicillin-resistant Staphylococcus aureus (MRSA), Stenotrophomonas maltophilia, Achromobacter xylosoxidans, and Haemophilus influenzae acquisition in young CF patients residing in the U.S. using the Cystic Fibrosis Foundation National Patient Registry, 2003-2009. Log-linear overdispersed Poisson regression was used to evaluate seasonal acquisition of each of these pathogens.

Results: A total of 4552 children met inclusion criteria. During follow-up 910 (20\%), 1161 (26\%), 228 (5\%), and 2148 (47\%) children acquired MRSA, S. maltophilia, A. xylosoxidans and H. influenzae, respectively. Compared to winter season, MRSA was less frequently acquired in spring (Incidence Rate Ratio [IRR]: 0.79; 95\% Confidence Interval [Cl]: 0.65, 0.96) and summer (IRR: $0.69 ; 95 \% \mathrm{Cl}: 0.57,0.84)$ seasons. Similarly, a lower rate of $A$. xylosoxidans acquisition was observed in spring (IRR: 0.59; $95 \% \mathrm{Cl}: 0.39$, 0.89). For $\mathrm{H}$. influenzae, summer (IRR: $0.88 ; 95 \% \mathrm{Cl}: 0.78,0.99$ ) and autumn (IRR: 0.78; $95 \%$ Cl: $0.69,0.88)$ seasons were associated with lower acquisition rates compared to winter. No seasonal variation was observed for S. maltophilia acquisition.

Conclusion: Acquisition of CF-related respiratory pathogens displays seasonal variation in young children with CF, with the highest rate of acquisition for most pathogens occurring in the winter. Investigation of factors underlying these observed associations may contribute to our understanding of the aetiology of these infections and guide future infection control strategies.
\end{abstract}

Keywords: Cystic fibrosis, Seasonality, MRSA, Stenotrophomonas maltophilia, Achromobacter xylosoxidans, Haemophilus influenzae

\footnotetext{
* Correspondence: kpsoter1@jhu.edu

'Department of Pediatrics, School of Medicine, The Johns Hopkins University

Bayview Medical Center, 5200 Eastern Ave, Mason F. Lord Bldg, Suite 4200,

Baltimore, MD 21224, USA

Full list of author information is available at the end of the article
} 


\section{Background}

Cystic fibrosis (CF), an autosomal recessive disorder, is characterized by chronic and recurring endobronchial infection, resulting in progressive structural lung disease and, generally, premature death. Beginning early in life, abnormal mucociliary clearance promotes colonization by a variety of bacteria $[1,2]$. Haemophilus influenzae and methicillin-susceptible Staphylococcus aureus (MSSA) are frequently among the first organisms isolated from respiratory cultures [1]; however, their effect on clinical outcomes is debated [2]. Pseudomonas aeruginosa, a Gram-negative bacterium frequently first isolated from respiratory cultures in early childhood, is regarded as the most important CF-related respiratory pathogen [3, 4]. Other gram-negative organisms and methicillin-resistant $S$. aureus (MRSA) often follow, and appear to also adversely affect clinical outcomes $[2,5]$.

While risk factors for specific bacterial infections have been reviewed $[2,5,6]$, little information is available regarding the incidence patterns of these infections. $P$. aeruginosa appears to be typically initially acquired from the natural environment; however, the sources for other organisms are far less clear. A more complete understanding of the incidence patterns of acquisition of other pathogens may provide an opportunity for the identification of potential aetiologic factors, as well as refinement of infection control strategies for these infections. Seasonal variation of infectious diseases is often observed [7] and increasing evidence suggests that many Gramnegative infections display such patterns $[8,9]$. Previously, we reported differential seasonal acquisition of initial $P$. aeruginosa in young children with CF and no such variation for MSSA infections [10]. The purpose of this study was to describe and compare seasonal incidence for other common CF-related respiratory bacterial pathogens including MRSA, Stenotrophomonas maltophilia, Achromobacter xylosoxidans and Haemophilus influenzae, in young CF patients residing in the U.S.

\section{Methods}

\section{Study population}

We performed a retrospective cohort study of the seasonal incidence of MRSA, S. maltophilia, A. xylosoxidans, and $H$. influenzae acquisition in U.S. children with $\mathrm{CF}<6$ years of age using the Cystic Fibrosis Foundation National Patient Registry, 2003-2009. The Registry contains detailed information on patient demographic and disease characteristics, including results of respiratory cultures at each clinical encounter [11]. Current clinical recommendations are for quarterly cultures (i.e. four times per year) [12]. Children $<6$ years of age typically do not expectorate sputum so the primary source of cultures in this age range is oropharyngeal swabs. Culturing for the infectious agents, and in the case of MRSA, antimicrobial resistance, is performed at the individual clinical microbiology laboratories and based on the selective media recommendations for each pathogen by the Cystic Fibrosis Foundation [13].

The study population consisted of children born after December 31, 2002. Only those children who: 1) were diagnosed with CF and 2) had a first respiratory culture performed prior to two years of age were included in the study cohort. This study was approved by the institutional review board at the University of Washington and the Cystic Fibrosis Foundation Registry Committee.

\section{Statistical analysis}

For each organism, demographic and disease characteristics were compared between those patients acquiring and remaining pathogen-free during follow-up using the Student $t$ test with unequal variances and the Chi square test for continuous and categorical variables, respectively.

Seasons were defined as: winter (December-February months), spring (March-May), summer (June-August) and autumn (September-November). Seasonal incidence for each of the respiratory pathogens was calculated over the six-year study period. The incident culture for each organism was defined as the first respiratory culture from which that organism was isolated as recorded in the Registry. Numerator data included the number of cases occurring during each season and the denominator was taken as the total person-seasons of children at risk for initial acquisition. Children entered the study upon first clinical encounter recorded in the Registry and remained at risk until either the pathogen was cultured or the study was completed. For those children in whom acquisition did not occur, the season in which the last clinical encounter was recorded was taken as the last season in which the child was at risk for acquisition.

Quasi-Poisson log-linear models with robust standard errors, modeled with the number of person-seasons of children at risk for acquisition as an offset term, were used to evaluate the seasonality of acquisition of each respiratory pathogen. Winter season served as the reference group for all analyses. Finally, we repeated the previously described analyses to evaluate whether seasonal patterns of acquisition differed within climate zones in the U.S. using the revised Köppen-Geiger climate classification [14] (Additional file 1). Results of regression analyses are reported as incidence rate ratios (IRR) and corresponding 95\% confidence intervals (CI). A twosided $P$ value of 0.05 was used to determine statistical significance. All analyses were conducted using the $R$ statistical environment (Version 3.0.2) [15].

\section{Results}

A total of 4522 children met inclusion criteria and were included in the study population. During follow-up a total of $910(20.1 \%)$ children acquired MRSA during 
55,629 person-seasons of follow-up, 1161 (25.7\%) children acquired S. maltophilia over 53,007 person-seasons, 228 (5.0\%) acquired A. xylosoxidans over 60,688 personseasons and $2148(47.5 \%)$ of the children acquired $H$. influenzae during 42,508 person-seasons of follow-up. Among those acquiring each organism, the median age at acquisition of MRSA, S. maltophilia, A. xylosoxidans and $H$. influenzae, respectively, was 25 months (25th-75th percentiles: 14-42 months), 21 (13-35), 32 (18-48) and 20 (13-31) months. Overall, approximately $55 \%$ of individuals had a culture frequency of four per year.

Table 1 describes the demographic and clinical characteristics of the study population by acquisition status of each pathogen. Children acquiring each of the organisms were less likely to have been identified by newborn screening than those remaining infection-free. Those acquiring S. maltophilia and A. xylosoxidans were more likely to be Hispanic than those remaining free of these organisms, while the converse was true for MRSA and $H$. influenzae. The mean age of CF diagnosis was greater among children acquiring MRSA, A. xylosoxidans and $H$. influenzae than among those remaining free of those pathogens. Finally, patients who acquired MRSA, S. maltophilia and $H$. influenzae were more likely to have CF mutations resulting in minimal CFTR function.

Incidence of each of the pathogens, overall and by season, are presented in Table 2. $H$. influenzae incidence
(50.5 per 1000 person-seasons) was highest overall, followed by A xylosoxidans (21.9 per 1000 personseasons), MRSA (16.4 per 1000 person-seasons), and $S$. maltophilia (3.8 per 1000 person-seasons). Peak incidence of MRSA (19.5 per 1000 person-seasons), A. xylosoxidans (4.9 per 1000 person-seasons), and H. influenzae (56.8 per 1000 person-seasons) was observed in the winter season, while S. maltophilia acquisition was highest in the summer season (23.6 per 1000 person-seasons).

Results of the Poisson regression evaluating the seasonality of each respiratory pathogen are presented in Table 3. Compared to winter, MRSA acquisition was less common in spring (IRR: 0.79; 95\% CI: $0.65,0.96$ ) and summer (IRR: 0.69; 95\% CI: 0.57, 0.84). A. xylosoxidans acquisition was also less likely in spring (IRR: 0.59; $95 \%$ CI: $0.39,0.89)$. H. influenzae acquisition was less likely in summer (IRR: $0.88 ; 95 \%$ CI: 0.78, 0.99) and autumn (IRR: 0.78 ; $95 \%$ CI: $0.69,0.88)$ seasons. No statistically significant seasonal differences were observed for S. maltophilia acquisition. Lastly, patterns of pathogen acquisition were similar within climate zones (Additional file 2).

\section{Discussion}

In this study, seasonal variation was observed for rates of initial acquisition of MRSA, A. xylosoxidans, and $H$. influenzae in young U.S. children with CF, while no such variation was observed for S. maltophilia acquisition.

Table 1 Demographic and clinical characteristics of young U.S. children with cystic fibrosis from 2003 to 2009, by pathogen acquisition status

\begin{tabular}{|c|c|c|c|c|c|c|c|c|}
\hline & \multicolumn{8}{|c|}{ Respiratory Pathogen } \\
\hline & \multicolumn{2}{|l|}{$\overline{M R S A}$} & \multicolumn{2}{|c|}{ S. maltophilia } & \multicolumn{2}{|c|}{ A. xylosoxidans } & \multicolumn{2}{|c|}{ H. influenzae } \\
\hline & $\begin{array}{l}\text { Acquired } \\
(n=910)\end{array}$ & $\begin{array}{l}\text { Negative } \\
(n=3612)\end{array}$ & $\begin{array}{l}\text { Acquired } \\
(n=1161)\end{array}$ & $\begin{array}{l}\text { Negative } \\
(n=3361)\end{array}$ & $\begin{array}{l}\text { Acquired } \\
(n=228)\end{array}$ & $\begin{array}{l}\text { Negative } \\
(n=4294)\end{array}$ & $\begin{array}{l}\text { Acquired } \\
(n=2148)\end{array}$ & $\begin{array}{l}\text { Negative } \\
(n=2374)\end{array}$ \\
\hline Male (\%) & $445(49 \%)$ & $1833(51 \%)$ & $590(51 \%)$ & $1688(50 \%)$ & $98(43 \%)$ & $2180(51 \%)$ & 1062(49\%) & $1216(51 \%)$ \\
\hline White (\%) & $825(91)$ & $3326(92)$ & 1065(92) & 3086(92) & 202(89) & 3949(92) & 1984(92) & 2167(91) \\
\hline Hispanic (\%) & $72(8)$ & $427(12)^{*}$ & $148(13)$ & $351(10)^{*}$ & $42(18)$ & $457(11)^{*}$ & 208(10) & $291(12)^{*}$ \\
\hline Identified by newborn screening (\%) & $280(31)$ & $1618(45)^{*}$ & $423(36)$ & $1475(44)^{*}$ & $73(32)$ & $1825(43)^{*}$ & $806(38)$ & $1092(46)^{*}$ \\
\hline Mean age at diagnosis, months (SD) & 2.9(4.6) & $2.4(4.3)^{*}$ & 2.6(4.3) & $2.4(4.4)$ & 3.5(5.3) & $2.4(4.3)^{*}$ & $2.7(4.5)$ & $2.3(4.2)^{*}$ \\
\hline \multicolumn{9}{|l|}{$\Delta$ F508 mutation category (\%) } \\
\hline Homozygous & 482(53) & $1553(43)^{*}$ & $586(50)$ & $1449(43)^{*}$ & $108(47)$ & 1927(45) & $996(46)$ & 1039(44) \\
\hline Heterozygous & $307(34)$ & 1437(40) & $390(34)$ & 1354(40) & $77(34)$ & 1667(39) & $802(37)$ & $942(40)$ \\
\hline Other & $79(9)$ & $463(13)$ & $132(11)$ & $410(12)$ & $32(14)$ & $510(12)$ & $255(12)$ & $287(12)$ \\
\hline \multicolumn{9}{|l|}{ CFTR functional class ${ }^{\mathrm{a}}(\%)$} \\
\hline Severe & $660(73)$ & $2284(63)^{*}$ & $817(70)$ & $2127(63)^{*}$ & $150(66)$ & $2794(65)$ & $1442(67)$ & $1502(63)^{*}$ \\
\hline Residual & $61(7)$ & $354(10)$ & $70(6)$ & $345(10)$ & $12(5)$ & 403(9) & 198(9) & $217(9)$ \\
\hline Unclassified & $189(21)$ & $974(27)$ & $274(24)$ & $889(26)$ & $66(29)$ & 1097(26) & $508(24)$ & $655(28)$ \\
\hline
\end{tabular}

MRSA, methicillin-resistant Staphylococcus aureus; S. maltophilia, Stenotrophomonas maltophilia; A. xylosoxidans, Achromobacter xylosoxidans; H. influenzae, Haemophilus influenzae; SD, standard deviation; CFTR, cystic fibrosis transmembrane conductance regulator

* $P<0.05$ based on Student's $t$ test with unequal variances for continuous variables or $x^{2}$ test for categorical variables.

a CFTR functional class is defined as follows: Severe, includes children in which CFTR mutations on both alleles result in minimal CFTR function (class 1, 2, or 3), including $\triangle \mathrm{F} 508$; Residual, at least one allele with a mutation resulting in partial CFTR function (class 4 or 5); Unclassified, both alleles with unknown functional class, or one allele with minimal CFTR function and the second with unknown functional class 
Table 2 Overall and seasonal acquisition and incidence (per 1000 person-seasons) of methicillin-resistant Staphylococcus aureus, Stenotrophomonas maltophilia, Achromobacter xylosoxidans, and Haemophilus influenzae among young U.S. children with cystic fibrosis, 2003 to 2009

\begin{tabular}{|c|c|c|c|c|c|c|c|c|c|c|}
\hline & & & Season & & & & & & & \\
\hline & Overall & & Winter & & Spring & & Summer & & Autumn & \\
\hline & $\overline{\text { Cases }(n)}$ & Incidence $^{a}$ & Cases (n) & Incidence $^{a}$ & $\overline{\text { Cases }(n)}$ & Incidence $^{a}$ & Cases (n) & Incidence $^{a}$ & $\overline{\text { Cases }(n)}$ & $\overline{\text { Incidence }}{ }^{a}$ \\
\hline MRSA & 910 & 16.4 & 238 & 19.5 & 207 & 15.4 & 195 & 13.5 & 270 & 17.4 \\
\hline S. maltophilia & 1161 & 21.9 & 263 & 22.4 & 259 & 20.3 & 324 & 23.6 & 315 & 21.3 \\
\hline A. xylosoxidans & 228 & 3.8 & 64 & 4.9 & 43 & 2.9 & 54 & 3.4 & 67 & 3.9 \\
\hline H. influenzae & 2148 & 50.5 & 563 & 56.8 & 524 & 52.2 & 537 & 50.0 & 534 & 44.4 \\
\hline
\end{tabular}

MRSA, methicillin-resistant Staphylococcus aureus; S. maltophilia, Stenotrophomonas maltophilia; A. xylosoxidans, Achromobacter xylosoxidans; H. influenzae, Haemophilus influenzae

${ }^{a}$ Per 1000 person-seasons

Compared to winter season, MRSA acquisition was less likely in spring and summer and A. xylosoxidans acquisition was less likely in spring. Summer and autumn seasons were associated with decreased $H$. influenzae acquisition compared to the winter season. Strengths of this investigation included a large national cohort of young children with $\mathrm{CF}$ with frequent monitoring of respiratory microbiology.

Interestingly, we previously reported seasonal variations in $P$. aeruginosa acquisition in the same cohort of young CF patients; however, the seasonal patterns of acquisition differed, with higher incidence of $P$. aeruginosa in summer (IRR: 1.22; 95\% CI: 1.07, 1.40) and autumn (IRR: 1.34; 95\% CI: 1.18, 1.52) seasons compared to winter [10]. Also, while in the current analyses we observed seasonal variation in MRSA acquisition, in our prior study no seasonal variation in MSSA acquisition was observed.

Table 3 Results of overdispersed Poisson log-linear regression evaluating seasonal incidence of methicillin-resistant Staphylococcus aureus, Stenotrophomonas maltophilia, Achromobacter xylosoxidans and Haemophilus influenzae acquisition among young U.S. children with cystic fibrosis, 2003 to 2009

\begin{tabular}{|c|c|c|c|c|}
\hline & \multicolumn{4}{|c|}{ Respiratory Pathogen } \\
\hline & MRSA & S. maltophilia & A. xylosoxidans & H. influenzae \\
\hline & $\begin{array}{l}\text { IRR } \\
(95 \% \mathrm{Cl})\end{array}$ & $\begin{array}{l}\text { IRR } \\
(95 \% \mathrm{Cl}) \\
\end{array}$ & $\begin{array}{l}\text { IRR } \\
(95 \% \text { CI) }\end{array}$ & $\begin{array}{l}\text { IRR } \\
(95 \% \mathrm{Cl}) \\
\end{array}$ \\
\hline Winter & $\begin{array}{l}1.0 \\
\text { Referent }\end{array}$ & $\begin{array}{l}1.0 \\
\text { Referent }\end{array}$ & $\begin{array}{l}1.0 \\
\text { Referent }\end{array}$ & $\begin{array}{l}1.0 \\
\text { Referent }\end{array}$ \\
\hline Spring & $\begin{array}{l}0.79^{*} \\
(0.65,0.96)\end{array}$ & $\begin{array}{l}0.91 \\
(0.76,1.08)\end{array}$ & $\begin{array}{l}0.59^{*} \\
(0.39,0.89)\end{array}$ & $\begin{array}{l}0.92 \\
(0.81,1.04)\end{array}$ \\
\hline Summer & $\begin{array}{l}0.69^{*} \\
(0.57,0.84)\end{array}$ & $\begin{array}{l}1.05 \\
(0.89,1.25)\end{array}$ & $\begin{array}{l}0.69 \\
(0.47,1.01)\end{array}$ & $\begin{array}{l}0.88^{*} \\
(0.78,0.99)\end{array}$ \\
\hline Autumn & $\begin{array}{l}0.89 \\
(0.75,1.07)\end{array}$ & $\begin{array}{l}0.95 \\
(0.80,1.12)\end{array}$ & $\begin{array}{l}0.80 \\
(0.56,1.15)\end{array}$ & $\begin{array}{l}0.78^{*} \\
(0.69,0.88)\end{array}$ \\
\hline
\end{tabular}

MRSA, methicillin-resistant Staphylococcus aureus; S. maltophilia, Stenotrophomonas maltophilia; A. xylosoxidans, Achromobacter xylosoxidans; H. influenzae, Haemophilus influenzae; IRR, incidence rate ratio; $\mathrm{Cl}$, confidence interval

${ }^{*} P<0.05$
The reason for these different seasonal patterns of pathogen acquisition is unclear. Seasonal factors can influence both host susceptibility and pathogen proliferation in the environment $[7,16-18]$. In CF patients, $P$. aeruginosa, a ubiquitous environmental organism, is typically acquired from the environment [19]. The source of other organisms is less clear but may include the indoor or outdoor environment, clinic or hospital settings and patient-to-patient transmission. We [20] and others [21] have shown that higher ambient temperatures increase the risk of $P$. aeruginosa acquisition among CF patients, which may partially explain our observation of a higher acquisition rate in the summer. On the other hand, viral respiratory infections, more prevalent in the winter both in CF patients and in the general population, are thought to increase airway susceptibility to bacterial colonization in CF patients [22-24], which may explain the higher observed rate of acquisition of MRSA, A. xylosoxidans and $H$. influenza in the winter months.

MRSA colonization is associated with poorer clinical outcomes in CF patients [25, 26] and is now frequently cultured from the respiratory tracts of patients. The overall prevalence among U.S. CF patients has risen from approximately $2 \%$ in 2001 to $25 \%$ in 2012 and even in young children $<2$ and 2-6 years MRSA prevalence in 2012 was 11 and 18\%, respectively [1]. In a multicenter study of the clonal distribution of MRSA isolates in children with CF, Champion and colleagues [27] reported that approximately $70 \%$ of all isolates were healthcare associated MRSA (HA-MRSA) strains. Further, recent studies have reported that HA-MRSA infections display a seasonal pattern in the general population, with peak rates occurring in winter months; this is in contrast to community acquired MRSA infections that peak in summer months $[28,29]$. In the current investigation, MRSA incidence was highest in the winter season as would be consistent with HA-MRSA infections typically found in this population. Interestingly, we observed no seasonal variation in MSSA acquisition among young CF patients in our prior study [10]; previous investigations of 
seasonal variations of nasal colonization of MSSA in the general population have reported mixed results [30].

Due to rare occurrences of S. maltophilia and A. xylosoxidans respiratory infections in the general population, evaluation of seasonal patterns has been limited and precludes comparison to results obtained herein. To our knowledge seasonal acquisition rates of these pathogens have not previously been described in the CF population. Accordingly, Burkholderia cepacia, a Gram-negative bacterium ubiquitous in the natural environment, is now recognized as a clinically significant pathogen in $\mathrm{CF}$ patients [2]. In this study meaningful analyses of B. cepacia could not be performed due to the relatively few cases $(n=41)$ of $B$. cepacia in this young population. During follow-up a total of $18,10,10$, and three cases of initial $B$. cepacia were reported in the spring, summer, autumn, and winter seasons, respectively.

The clinical significance of first isolation of bacterial pathogens from respiratory cultures in young CF patients is a topic of debate and likely varies by organism [5]. While initial $P$. aeruginosa colonization can spontaneously clear, it is likely to develop into chronic infection, which is nearly impossible to eradicate and is associated with poorer outcome and survival $[31,32]$. Thus standard of care around the world is to attempt to eradicate initial $P$. aeruginosa regardless of symptoms. For other organisms, the natural history is not well described and antibiotic treatment decisions are not standardized [33, 34]. Nonetheless, a potential "window of opportunity" during seasons of higher risk may present an opportunity for more intensive monitoring or targeted interventions to minimize infection risk.

There are several limitations to the present investigation. First, the exact date of pathogen acquisition was unknown due to the non-acute, subclinical nature of these infections. In this study, culturing was generally performed at quarterly intervals that enabled a "natural" seasonal analysis of incidence patterns. Second, differentiation between subtypes of bacteria species could not be performed in this study. For example, the Registry does not contain information regarding SCCmec types or Panton Valentine leukocidin genes with which to differentiate community and healthcare-associated MRSA. Given trends in the general population, it is possible that seasonal acquisition differs by subtype. Similarly, $H$. influenzae subtypes data were not collected so seasonal patterns of these subtypes could not be evaluated. Third, our study was limited to children diagnosed with $\mathrm{CF}$ prior to two years of age with a maximum of six years follow-up; therefore the generalizability of results is limited to very young children. Fourth, data on viral infections was unavailable in the Registry and may play an important role in viral induced secondary bacterial infections resulting from respiratory tract damage. Finally, the majority of pathogens were identified through culturing of isolates obtained from oropharyngeal swabs. Rosenfeld and colleagues [35] previously reported a moderate sensitivity and specificity for identifying $P$. aeruginosa and MSSA by oropharyngeal swab compared to bronchoalveolar lavage in children with CF; however, the accuracy of oropharyngeal cultures for the presence of the pathogens evaluated herein in the lower respiratory tract is unknown. Therefore, results from this study more accurately reflect upper airway than lower airway pathogen acquisition.

\section{Conclusions}

In conclusion, seasonal variations of bacterial respiratory pathogen acquisition rates were observed in a large cohort of young children with CF residing in the U.S. Future studies of factors, such as macroenvironmental variables, that may be contributing to these observed incidence patterns are needed. Similarly, the impact of global warming on both local weather patterns and extreme weather events may impact seasonal acquisition of respiratory pathogens. These findings may then contribute to our understanding of the aetiology of these infections and may provide guidance for future infection control strategies.

\section{Additional files}

\section{Additional file 1: Methods (DOCX $14 \mathrm{~kb}$ )}

Additional file 2: Seasonal incidence of methicillin-resistant Staphylococcus aureus, Stenotrophomonas maltophilia, Achromobacter xylosoxidans and Haemophilus influenzae acquisition among children $<6$ years of age with cystic fibrosis in the United States from 2003 to 2009, based upon Poisson regression models with the number of individuals at risk as the offset term and winter season as the season of reference (DOCX $17 \mathrm{~kb}$ )

\section{Abbreviations \\ A. xylosoxidans: Achromobacter xylosoxidans; B. cepacia: Burkholderia cepacia: CF: Cystic fibrosis; CFTR: Cystic fibrosis transmembrane conductance regulator; $\mathrm{Cl}$ : Confidence interval; $H$. influenzae: Haemophilus influenzae; IRR: Incidence rate ratio; MRSA: Methicillin-resistant Staphylococcus aureus; MSSA: Methicillin-susceptible Staphylococcus aureus; P. aeruginosa: Pseudomonas aeruginosa; S. maltophilia: Stenotrophomonas maltophilia; SD: Standard deviation}

\section{Acknowledgements \\ The authors would like to thank the Cystic Fibrosis Foundation for the use of CF Foundation Patient Registry data to conduct this study. Additionally, we would like to thank the patients, care providers, and clinic coordinators at CF Centers throughout the United States for their contributions to the CF Foundation Patient Registry.}

\section{Funding}

No funding was received at any stage for this study.

Availability of data and materials

The data that support the findings of this study are available from the Cystic Fibrosis Foundation but restrictions apply to the availability of these data, which were used under license for the current study, and so are not publicly available. 


\section{Authors' contributions}

All of the authors had access to the full dataset (including the statistical reports and tables) and take responsibility for the integrity of the data and the accuracy of the data analysis. KJP, AJD, JW, JDM, MR conceived and designed the study. KJP and MR collected the data and KJP analyzed the data. KJP, AJD, JW, JDM, MR interpreted the data. KJP and MR wrote the first draft of the paper. KJP, AJD, JW, JDM, MR reviewed and approved the final report.

\section{Competing interests}

The authors declare that they have no competing interests.

\section{Consent for publication}

Not applicable.

\section{Ethics approval and consent to participate}

This study was approved by the institutional review board at the University of Washington and the Cystic Fibrosis Foundation Registry Committee.

\section{Publisher's Note}

Springer Nature remains neutral with regard to jurisdictional claims in published maps and institutional affiliations.

\section{Author details}

'Department of Pediatrics, School of Medicine, The Johns Hopkins University Bayview Medical Center, 5200 Eastern Ave, Mason F. Lord Bldg, Suite 4200, Baltimore, MD 21224, USA. ${ }^{2}$ Department of Environmental and Occupational Health, Drexel University School of Public Health, Philadelphia, PA, USA. ${ }^{3}$ Departments of Biostatistics and Statistics, University of Washington, Seattle, WA, USA. ${ }^{4}$ Departments of Epidemiology, Geography, Global Health, Medicine (Allergy and Infectious Diseases), Family Medicine, and Health Services, University of Washington, Seattle, WA, USA. ${ }^{5}$ Division of Pulmonary Medicine, University of Washington School of Medicine, Seattle, WA, USA. ${ }^{6}$ Department of Pediatrics, Seattle Children's Hospital, Seattle, WA, USA.

Received: 8 November 2016 Accepted: 1 June 2017

Published online: 09 June 2017

\section{References}

1. Cystic Fibrosis Foundation. Patient registry 2012 annual report. Bethesda: Cystic Fibrosis Foundation; 2013.

2. Lipuma JJ. The changing microbial epidemiology in cystic fibrosis. Clin Microbiol Rev. 2010;23:299-323.

3. Emerson J, Rosenfeld M, McNamara S, Ramsey B, Gibson RL. Pseudomonas Aeruginosa and other predictors of mortality and morbidity in young children with cystic fibrosis. Pediatr Pulmonol. 2002;34:91-100.

4. Nixon GM, Armstrong DS, Carzino R, Carlin JB, Olinsky A, Robertson CF, et al. Clinical outcome after early Pseudomonas Aeruginosa infection in cystic fibrosis. J Pediatr. 2001:138:699-704

5. Hauser AR, Jain M, Bar-Meir M, McColley SA. Clinical significance of microbial infection and adaptation in cystic fibrosis. Clin Microbiol Rev. 2011;24:29-70.

6. Ciofu $\mathrm{O}$, Hansen $\mathrm{CR}$, Hoiby N. Respiratory bacterial infections in cystic fibrosis. Curr Opin Pulm Med. 2013;19:251-8.

7. Fisman DN. Seasonality of infectious diseases. Annu Rev Public Health 2007;28:127-43.

8. Richet $\mathrm{H}$. Seasonality in gram-negative and healthcare-associated infections. Clin Microbiol Infect. 2012;18:934-40.

9. Freeman J, Anderson D, Sexton DJ. Emerging evidence for seasonality of gramnegative bacterial infections. Infect Control Hosp Epidemiol. 2009;30:813-4.

10. Psoter KJ, De Roos AJ, Wakefield J, Mayer J, Rosenfeld M. Season is associated with Pseudomonas Aeruginosa acquisition in young children with cystic fibrosis. Clin Microbiol Infect. 2013;19:E483-9.

11. Knapp EA, Fink AK, Goss CH, Sewall A, Ostrenga J, Dowd C, et al. The Cystic Fibrosis Foundation patient registry. Design and methods of a National Observational Disease Registry. Ann Am Thorac Soc. 2016;13:1173-9.

12. Cystic Fibrosis Foundation, Borowitz D, Robinson KA, Rosenfeld M, Davis SD, Sabadosa KA, et al. Cystic Fibrosis Foundation evidence-based guidelines for management of infants with cystic fibrosis. J Pediatr. 2009;155:S73-93.

13. Saiman L, Siegel J, Cystic FF. Infection control recommendations for patients with cystic fibrosis: microbiology, important pathogens, and infection control practices to prevent patient-to-patient transmission. Infect Control Hosp Epidemiol. 2003;24:S6-52.
14. Peel MC, Finlayson BL, McMahon TA. Updated world map of the KoppenGeiger climate classification. Hydrol Earth Syst Sci. 2007;11:1633-44.

15. R Development Core Team. R: a language and environment for statistical computing. Vienna: R Foundation for Statistical Computing; 2013.

16. Naumova EN. Mystery of seasonality: getting the rhythm of nature. J Public Health Policy. 2006:27:2-12.

17. Dowell SF. Seasonal variation in host susceptibility and cycles of certain infectious diseases. Emerg Infect Dis. 2001;7:369-74.

18. Nelson RJ, Drazen DL. Melatonin mediates seasonal adjustments in immune function. Reprod Nutr Dev. 1999;39:383-98.

19. Kidd TJ, Ramsay KA, Vidmar S, Carlin JB, Bell SC, Wainwright CE, et al. Pseudomonas Aeruginosa genotypes acquired by children with cystic fibrosis by age 5-years. J Cyst Fibros. 2015;14:361-9.

20. Psoter KJ, De Roos AJ, Wakefield J, Mayer JD, Bryan M, Rosenfeld M. Association of meteorological and geographical factors and risk of initial Pseudomonas Aeruginosa acquisition in young children with cystic fibrosis. Epidemiol Infect. 2016;144:1075-83.

21. Collaco JM, McGready J, Green DM, Naughton KM, Watson CP, Shields T, et al. Effect of temperature on cystic fibrosis lung disease and infections: a replicated cohort study. PLoS One. 2011;6:e27784

22. Hament JM, Kimpen JL, Fleer A, Wolfs TF. Respiratory viral infection predisposing for bacterial disease: a concise review. FEMS Immunol Med Microbiol. 1999;26:189-95.

23. Ortiz JR, Neuzil KM, Victor JC, Wald A, Aitken ML, Goss CH. Influenzaassociated cystic fibrosis pulmonary exacerbations. Chest. 2010;137:852-60.

24. Somayaji R, Goss CH, Khan U, Neradilek M, Neuzil KM, Ortiz JR. Cystic fibrosis pulmonary exacerbations attributable to respiratory syncytial virus and influenza: a population-based study. Clin Infect Dis. 2017;In Press.

25. Ren CL, Morgan WJ, Konstan MW, Schechter MS, Wagener JS, Fisher KA, et al. Presence of methicillin resistant Staphylococcus aureus in respiratory cultures from cystic fibrosis patients is associated with lower lung function. Pediatr Pulmonol. 2007:42:513-8.

26. Sawicki GS, Rasouliyan $L$, Ren $C L$. The impact of MRSA on lung function in patients with cystic fibrosis. Am J Respir Crit Care Med. 2009;179:734-5.

27. Champion EA, Miller MB, Popowitch EB, Hobbs MM, Saiman L, Muhlebach MS, et al. Antimicrobial susceptibility and molecular typing of MRSA in cystic fibrosis. Pediatr Pulmonol. 2014;49:230-7.

28. Mermel LA, Machan JT, Parenteau S. Seasonality of MRSA infections. PLoS One. 2011:6:e17925

29. Klein EY, Sun L, Smith DL, Laxminarayan R. The changing epidemiology of methicillin-resistant Staphylococcus aureus in the United States: a national observational study. Am J Epidemiol. 2013:177:666-74.

30. Leekha S, Diekema DJ, Perencevich EN. Seasonality of staphylococcal infections. Clin Microbiol Infect. 2012;18:927-33.

31. Henry RL, Mellis CM, Petrovic L. Mucoid Pseudomonas Aeruginosa is a marker of poor survival in cystic fibrosis. Pediatr Pulmonol. 1992;12:158-61.

32. Schaedel C, de Monestrol I, Hjelte L, Johannesson M, Kornfalt R, Lindblad A, et al. Predictors of deterioration of lung function in cystic fibrosis. Pediatr Pulmonol. 2002;33:483-91.

33. Chmiel JF, Berger M, Konstan MW. The role of inflammation in the pathophysiology of CF lung disease. Clin Rev Allergy Immunol. 2002;23:5-27.

34. Doring G, Flume P, Heijerman H, Elborn JS, Consensus SG. Treatment of lung infection in patients with cystic fibrosis: current and future strategies. J Cyst Fibros. 2012;11:461-79.

35. Rosenfeld M, Emerson J, Accurso F, Armstrong D, Castile R, Grimwood K, et al. Diagnostic accuracy of oropharyngeal cultures in infants and young children with cystic fibrosis. Pediatr Pulmonol. 1999:28:321-8. 